\title{
Hysteroscopic Endometrial Polypectomy: Comparative Retrospective Study of the Morcellator System versus Electrosurgical Resection
}

\author{
José Manuel López-Carral ${ }^{1 *}$, Ana Fernández Novo ${ }^{1}$, Andrea Fernández Iglesias ${ }^{1}$ and Pablo Martin- \\ Lancharro ${ }^{2}$
}

${ }^{1}$ Department of Obstetrics and Gynecology, University Hospital of Santiago de Compostela, Spain

${ }^{2}$ Department of Statistics, University Hospital of Santiago de Compostela, Spain

\begin{abstract}
Objective: To evaluate the differences observed between hysteroscopic morcellation and electrosurgical resection in patients with endometrial polyps in a tertiary hospital.

Patients and methods: A single-center observational retrospective comparative study was performed, including 190 patients who underwent hysteroscopic resection of endometrial polyps from January 2017 to December 2017. The hysteroscopic techniques were TRUCLEAR $5.5^{\circledR}$ to morcellation and Karl Storz ${ }^{\circledR}$ monopolar resection.

Results: Eighty-seven patients underwent resection of the polyp morcellation with TRUCLEAR $5.5^{\circledR}$ and 103 with monopolar resection Karl Storz ${ }^{\circledast}$. Surgical time (mean) was 11.86 minutes in the morcellation group and 15.39 minutes in the monopolar resection group $(\mathrm{P}<0.001)$. Size of polyps were $1.45 \mathrm{~cm}$ in the morcellation group compared to $1.32 \mathrm{~cm}$ in the resection group $(P=0.203)$. No significant differences were observed due to location and number of polyps. The polyps were completely removed in $100 \%$ of cases in both groups. No complications in terms of uterine perforation and cervical laceration or fluid deficit were observed in the two groups.
\end{abstract}

Discussion and conclusion: The hysteroscopic morcellation technique was significantly faster and equally effective by completely removing endometrial polyps compared to electrosurgical resection. No differences were observed in size, location and number of polyps between two groups.

Keywords

Hysteroscopy, Polyps, Hysteroscopic morcellation, Monopolar resectoscopy

\section{Introduction}

Endometrial polyps justify a large part of dysfunctional bleeding in fertile women and an important part of metrorrhagia in postmenopausal patients [1-3]. For its treatment, endoscopic techniques are increasingly common in the world of minimally invasive gynaecological surgery. Its numerous advantages, focused on the reduced time of the intervention and the rapid recovery to the process, together with the great advances in the operative instruments, have made hysteroscopy a widespread technique nowadays. The most common procedure consists of the exeresis of endometrial polyps [4], called "see and treat", because in the same surgical act the existence of the polyp is confirmed and then the polypectomy is made, as a unique surgical act.

A recent technique in surgical hysteroscopy is hysteroscopic morcellation, as an effective and safe alternative to electrosurgical resection [5]. The first study about the use of the morcellator was published by Emanuel, et al. where they recorded a significant reduction in surgical time in polypectomies and resections of myomas type I and II with morcellator, compared to the traditional resector [6]. Hysteroscopic morcellation consists of a rigid internal tube that rotates inside another outer tube and combines cutting and aspiration. The

*Corresponding author: José Manuel López-Carral, PhD, MD, Department of Obstetrics and Gynecology, University Hospital of Santiago de Compostela, Trav. Choupana, s/n, 15706 Santiago de Compostela, Spain, Tel: +34-981-950236

Accepted: September 12, 2019

Published online: September 14, 2019

Citation: López-Carral JM, Novo AF, Iglesias AF, et al. (2019) Hysteroscopic Endometrial Polypectomy: Comparative Retrospective Study of the Morcellator System versus Electrosurgical Resection. Reports Gynecol Surg 2(1):22-26 
Citation: López-Carral JM, Novo AF, Iglesias AF, et al. (2019) Hysteroscopic Endometrial Polypectomy: Comparative Retrospective Study of the Morcellator System versus Electrosurgical Resection. Reports Gynecol Surg 2(1):22-26

aspiration system is connected to the morcellator itself and the material obtained is collected in an easily removable bag to be sent to a later anatomopathological study. Saline solution is used as an irrigating solution [6].

It has been demonstrated that the use of a non-electrolytic/non-conductive liquid distension medium (glycine) can produce aqueous intoxication in case of massive absorption into the bloodstream, when the monopolar resectoscope is used. On the other hand, the use of sodium chloride $0.9 \%$ can produce water overload in the case of important intravasation, but it does not occur with hyponatremia $[2,3]$.

It is important to see that the morcellator facilitates the intervention because it improves the visualization during the surgery when the tissue fragments, which are generated during the resection of the endometrial formation, are aspirated. On the other hand the electrosurgical resection requires the periodic extraction of the resected material, which in addition to increasing the surgical time also increases the risk of uterine perforation due to poorer visualization of the surgical field $[2,4,7]$.

In relation to the morcellator, a differentiating aspect is that it allows an intervention without general anaesthesia, unlike the traditional technique with electrosurgical resection that requires previous cervical dilatation, for which anaesthesia is necessary. For this reason, morbidity is also reduced with the use of the morcellator since most of the patients do not require prior preparation and therefore manipulation during the surgical act is reduced $[2,4]$.

The objective of this study was to compare the safety and frequency of adverse effects, surgical time according to the intervention conditions and success in hysteroscopic polypectomy using hysteroscopic morcellation versus electrosurgical resection in the population of a tertiary university hospital.

\section{Material and Methods}

This study was designed according to the Declaration of Helsinki. All the participants were selected from patients treated by the Gynaecology and Obstetrics Service of the Hospital Clínico de Santiago and the informed consent for inclusion in the study was obtained in the post-surgery examination. The methodological design selected was a single-center observational retrospective comparative study.

The study period was from January 2017 to December of the same year (12 months). The inclusion criteria were all those adult women attended in gynaecological practice with suspected ultrasound of an endometrial polyp, metrorrhagia or thickened endometrium, regardless of the size or number of polyps for ultrasound scan. The exclusion criteria were all those patients whose submucosal myoma was evidenced during the hysteroscopy, ultrasound or anatomopathological suspicion of malignancy; contraindication of surgical hysteroscopy; pregnancy; or active pelvic surgical infection.

Interventions were performed by a single specialist in gynaecology and obstetrics. The gynaecologist had sufficient experience in both methods of hysteroscopic polypectomy, although he had greater experience with the electrosurgical resection technique because morcellation is a more recent method.

Interventions were performed in an operating theatre in the Major Ambulatory Surgery Unit. Admission to the Unit occurred on the same morning, followed by a review of the patient's clinical history and a pregnancy test in all patients of childbearing age. The anaesthesia administered to each patient was at the discretion of each anaesthesiologist, who used a lower anaesthetic depth in patients who underwent resection with a morcellator. None of the patients received antibiotic prophylaxis.

Vaginoscopy, understood as a step from the hysteroscope to the uterine cavity without the use of a vaginal speculum, was the chosen method to entry into the cavity during interventions. After the surgery, patients were discharged on the same day of the surgery at 6 hours after the intervention, except in the case of complications. The appointment for next revision was 4 weeks after the operation.

Glycine was used as the fluid for the electrosurgical technique whereas sodium chloride $0.9 \%$ was used for the morcellation technique.

The criteria for choosing one polypectomy technique or another was the availability of the tissue shaver device (that is inserted through a hysteroscope morcellator to simultaneously cut and aspirate tissue) on the day of the intervention. Since the morcellator is more expensive technique, it is not always available for use. The hysteroscopes used were rigid electrosurgical resector Karl Storz ${ }^{\circledR}$ hysteroscope of 7 and 9 $\mathrm{mm}$, monopolar with 30-degree optic (Figure 1) and hysteroscope morcellator TRUCLEAR ${ }^{\circledR} 5.5$ system with 0 -degree optics (Figure 2). Pressures and flows used during the intervention varied according to the technique. Flows of up to 400 $\mathrm{mL} / \mathrm{min}$ were handled in case of electrosurgical resector and maximum pressures of $150 \mathrm{mmHg}$. In case of morcellator hysteroscope, maximum flows of $250 \mathrm{ml} / \mathrm{min}$ and pressures of up to $80 \mathrm{mmHg}$ were used. In morcellator interventions, the direction of the internal tube is also regulated, which can be oscillatory or continuous and in polypectomies, oscillatory mode was chosen at 1500 rotations per minute.

Variables used for the study were: age; number of children; previous caesareans; menopausal state, defined as pre or postmenopausal; preoperative diagnosis (polyp suspicion, metrorrhagia or thickened endometrium); surgical time (time elapsed between the insertion in the vagina until the exit thereof); number of polyps; size of the polyps; location (anterior, posterior, fundus and lateral); success, understood as the complete resection of all polyps; adverse effects, such as vaginal bleeding, infection, uterine perforation or fluid overload due to absorption of the distension medium.

In terms of statistical analysis, demographic and clinical variables of the study population were analyzed descriptively. For continuous variables, mean and standard deviation (SD) were calculated for each type of hysteroscopy technique, and number and percentage were calculated for categorical variables. Differences between surgical times and polyp size for 
Citation: López-Carral JM, Novo AF, Iglesias AF, et al. (2019) Hysteroscopic Endometrial Polypectomy: Comparative Retrospective Study of the Morcellator System versus Electrosurgical Resection. Reports Gynecol Surg 2(1):22-26

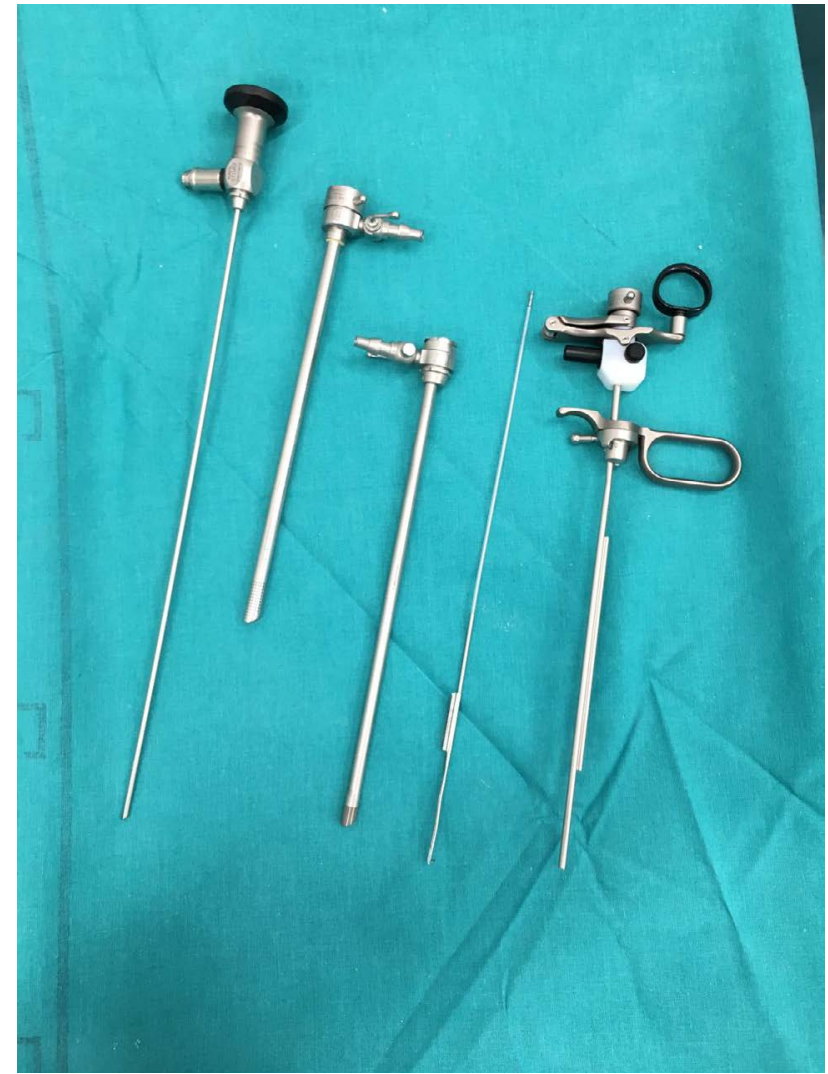

Figure 1: Karl Stor $z^{\circledR}$ hysteroscope of 7 and $9 \mathrm{~mm}$, monopolar with 30 degree optic.

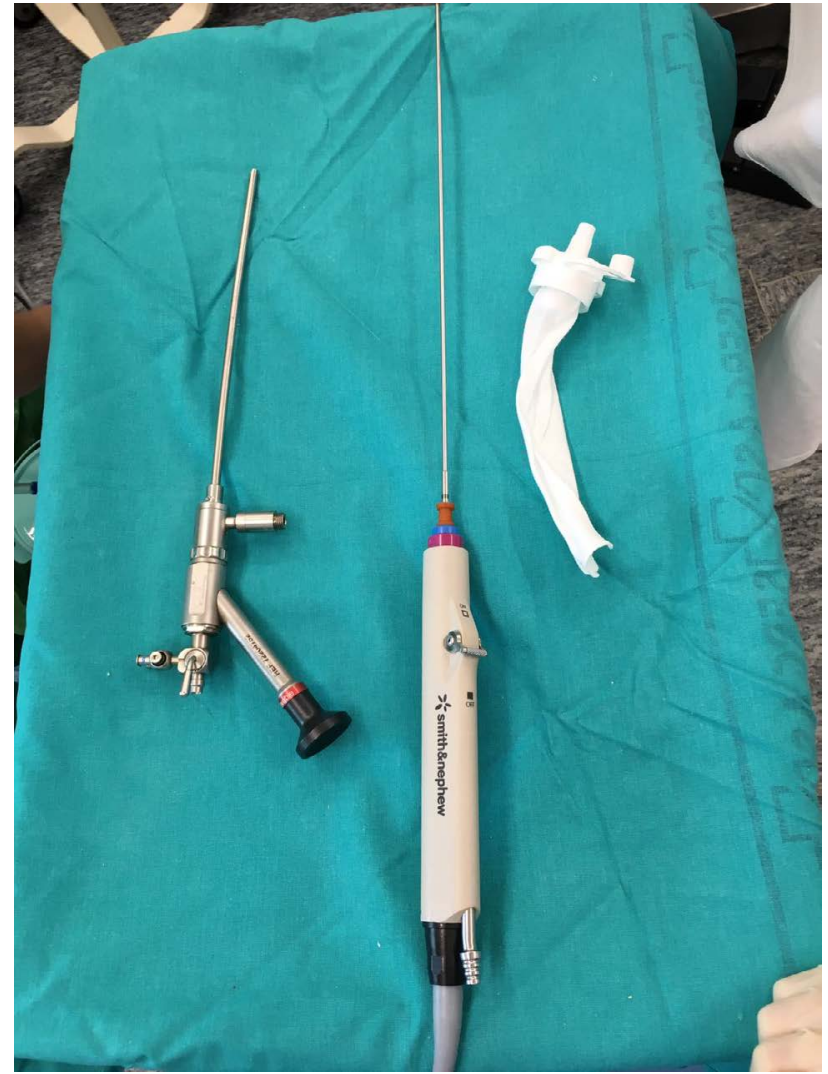

Figure 2: $\operatorname{TRUCLEAR}^{\circledR} 5.5$ hysteroscope morcellator.

Table 1: Demographic and clinical characteristics of the study population.

\begin{tabular}{|l|l|l|}
\hline Surgical technique & Morcellator $(\mathbf{n}=\mathbf{8 7})$ & Resector $(\mathbf{n}=\mathbf{1 0 3})$ \\
\hline Age (years \pm SD) & $48.60 \pm 9.78$ & $49.23 \pm 9.53$ \\
\hline Children (mean \pm SD) & $1.20 \pm 1.10$ & $1.48 \pm 1.16$ \\
\hline Previous caesareans (mean \pm SD) & $0.13 \pm 0.45$ & $0.17 \pm 0.46$ \\
\hline Menopausal state & $61(70.1 \%)$ & $68(66.0 \%)$ \\
\hline Pre & $26(29.9 \%)$ & $35(34.0 \%)$ \\
\hline Post & & \\
\hline Preoperative diagnosis & $79(90.8 \%)$ & $94(91.2 \%)$ \\
\hline Polyp suspicion & $4(4.6 \%)$ & $5(4.9 \%)$ \\
\hline Metrorrhagia & $4(4.6 \%)$ & $4(3.9 \%)$ \\
\hline Thickened endometrium & & \\
\hline
\end{tabular}

each type of hysteroscopy technique were compared using the t-Student test. Location and number of polyps was compared using the $\chi^{2}$ test. Comparison between success and adverse effects was made by Fisher's exact test. All analyses were performed using SPSS software, Version 20.0 (SPSS Inc., Chicago, IL, USA). Differences at $p<0.05$ were considered statistically significant.

\section{Results}

190 patients were included in the retrospective study. These patients underwent the removal of one or more endometrial polyps as part of their standard care at the
Hospital Clínico Universitario de Santiago between January and December 2017.

Most of the variables that characterize the population under study, demographic and clinical, did not show significant differences between patients who underwent electrosurgical resection or hysteroscopic morcellation (Table 1 ), so there are two homogeneous groups. However, we emphasize that women undergoing hysteroscopic morcellation had fewer children and previous caesarean sections on average than those operated on by electrosurgical resection.

The mean time required to complete the polypectomy 
Citation: López-Carral JM, Novo AF, Iglesias AF, et al. (2019) Hysteroscopic Endometrial Polypectomy: Comparative Retrospective Study of the Morcellator System versus Electrosurgical Resection. Reports Gynecol Surg 2(1):22-26

procedure was $11.86 \pm 4.10$ minutes for hysteroscopic morcellation compared to $15.39 \pm 5.52$ minutes for electrosurgical resection $(p<0,001)$. Regarding the mean size of the polyps, the hysteroscopic morcellation group was $1.45 \pm 0.77 \mathrm{~cm}$ compared to $1.32 \pm 0.66 \mathrm{~cm}$ of the electrosurgical resection group ( $p<0.203$ ) (Table 2).

Complete removal of polyps was achieved in 87 of 87 (100\%) patients with hysteroscopic morcellation and 103 of $103(100 \%)$ patients who underwent electrosurgical resection $(p=0.999)$ (Table 2). The use of either technique did not lead to differences in terms of success.

Location was different in both groups, with a greater number of cases located in the fundus for hysteroscopic morcellation (41.4\%) compared to electrical resection (32\%), but lower in cases of lateral and posterior situation $(24.1 \%$ and $21.8 \%)$, than for electrosurgical resection $(29.1 \%$ and $25.2 \%$ respectively). The location between both groups did not show significant differences $(p=0.559)$ (Table 2$)$.

The number of polyps extracted in each surgery showed differences between hysteroscopic morcellation and electrical resection. Patients with 1 polyp were $77.7 \%$ for electrical resection and $69 \%$ for morcellation. The patients with 3 or 4 polyps were $6.8 \%$ for hysteroscopic morcellation versus the $1.9 \%$ of the patients who underwent electrosurgical resection. These differences between the groups did not show significant differences $(p=0.281)$ (Table 2$)$.

The polyps removed were those symptomatic polyps of any size and those asymptomatic polyps bigger than $15 \mathrm{~mm}$.

There were no adverse events in the 190 patients due to intrauterine injury or fluid deficit.

\section{Discussion and Conclusion}

In this retrospective study conducted in a hospital that attends a large number of patients, we compared the 2 hysteroscopy methods available in the Obstetrics and Gynecology Department, and observed a $29 \%$ increase in the intervention time with the electrical resection. This finding can be explained as the hysteroscopic morcellation performs the cutting and extraction of tissue simultaneously through the active suction device. The simple configuration for hysteroscopic morcellation procedure can further reduce the time needed to perform the procedure by decreasing the installation time.

Our study confirmed the results on the resection of endometrial polyps previously published $[3,4,8,9]$. Hamerlynck, et al. [3] which prospectively compared the morcellator vs. the resector and found a reduction in total time of operation for the removal of endometrial polyps by hysteroscopic morcellation (average time $9.5 \mathrm{~min}$ compared with $12.2 \mathrm{~min}$ ). Pampalona, et al. [10] found a reduction in average operating time when comparing hysteroscopic morcellation using the TRUCLEAR 5.0 system versus bipolar resectoscopy GYNECARE VERSAPOINT (6.82 min compared with $11.62 \mathrm{~min}$ ). Tsuchiya, et al. [8] also found a reduction in operating time $(8.3 \mathrm{~min}$ compared with $12.0 \mathrm{~min}$ ), similar to the other studies mentioned above.

In the retrospective studies published by Hamidouche, et al. [11] and Emanuel, et al. [6], shorter operation times were observed for hysteroscopic morcellation (mean $16.4 \pm 6.5$ min compared to $17.8 \mathrm{~min} \pm 10.8$, and a mean of $8.7 \pm 3.5 \mathrm{~min}$ compared with $30.9 \pm 12.9$ min, respectively). As our study is retrospective, this presents a limitation against the data of

Table 2: Characteristics of polyps and surgical procedure.

\begin{tabular}{|c|c|c|c|}
\hline Surgical technique & Morcellator $(n=87)$ & Resector $(n=103)$ & $P$ value \\
\hline Pólyps size (cm) & $1.45 \pm 0.77$ & $1.32 \pm 0.66$ & 0.203 \\
\hline Surgical time $\left(n^{\circ}\right)$ & $11.86 \pm 4.10$ & $15.39 \pm 5.52$ & $<0.001$ \\
\hline \multicolumn{3}{|l|}{ Location } & 0.559 \\
\hline Anterior & $11(12.6 \%)$ & $14(13.6 \%)$ & \\
\hline Fundus & $36(41.4 \%)$ & $33(32.0 \%)$ & \\
\hline Lateral & $21(24.1 \%)$ & 30 (29.1\%) & \\
\hline Posterior & $19(21.8 \%)$ & $26(25.2 \%)$ & \\
\hline \multicolumn{3}{|l|}{ Polyps $\left(n^{\circ}\right)$} & 0.281 \\
\hline 1 & $60(69.0 \%)$ & $80(77.7 \%)$ & \\
\hline 2 & $21(24.1 \%)$ & $21(20.4 \%)$ & \\
\hline 3 & $5(5.7 \%)$ & $2(1.9 \%)$ & \\
\hline 4 & $1(1.1 \%)$ & $0(0 \%)$ & \\
\hline \multicolumn{3}{|l|}{ Success } & 0.999 \\
\hline Total & 87 (100\%) & $103(100 \%)$ & \\
\hline Partial & $0(0 \%)$ & $0(0 \%)$ & \\
\hline \multicolumn{3}{|l|}{ Adverse effects } & 0.999 \\
\hline Yes & $0(0 \%)$ & $0(0 \%)$ & \\
\hline No & 87 (100\%) & $103(100 \%)$ & \\
\hline
\end{tabular}


Citation: López-Carral JM, Novo AF, Iglesias AF, et al. (2019) Hysteroscopic Endometrial Polypectomy: Comparative Retrospective Study of the Morcellator System versus Electrosurgical Resection. Reports Gynecol Surg 2(1):22-26

the prospective RCT.

In our study, the average size of the operated polyps (1.45 and 1.32 for morcellator and resector respectively) was similar to other studies (1.6 and 1.5 by Hamerlynck, et al. [3]; and 1.7 by Smith, et al. [4]). Only the study by Hamidouche, et al. [11] showed smaller sizes, probably due to the low number of patients included in the study $(25$ patients).

In relation to the number of polyps present in each patient, our study showed values $(1.39 \pm 0.65$ and $1.24 \pm 0.47$ for morcellator and resector respectively) that coincide with the rest of the studies even though they are slightly lower: 1.8 \pm 0.9 and $1.2 \pm 0.5$ for morcellator and resector in Smith, et al. [4]; $1.6 \pm 1.1$ and $1.8 \pm 1.0$ in Emanuel, et al. [6]. However, the percentage of cases with more than 1 polyp in the morcellator group was higher than the resector group (31\% versus $22.3 \%)$, which could influence the intervention time used, although the differences were not significant $(p=0.281)$.

The polyp location was $41.4 \%$ for morcellator and $32 \%$ for resector in relation to the fundal position that allows comparison with other studies. These values are lower than those shown by the study by Pampalona, et al. [10] and Hamerlynck, et al. [3], but more similar to the study by Smith, et al. [4], with $31 \%$ and $27 \%$ for morcellator and resector respectively.

We have not detected significant differences in the adverse effects derived from the intervention ( $p<0.999)$, such as perforation risk, cervical laceration, neurological toxicity of glycine or fluid deficit. However, we believe that hysteroscopic morcellation may decrease perforation risk related to reintroduction as a result of immediate tissue removal, with fewer hysteroscopic re-insertions and good visibility, which is reflected in the longer intervention time for electrosurgical resection. In the study by Hamerlynck, et al. [7] 4 cases of perforation were observed ( 1 in hysteroscopic morcellation and 3 in electrosurgical resection) in 84 patients.

In relation to success, no differences were observed between the 2 groups ( $p<0.999$ ), like Tsuchiya, et al. [8], Smith, et al. [4] and Pampalona, et al. [10] showed a higher rate of complete elimination of polyps in the outpatient hysteroscopic morcellation group ( $98 \%$ versus $83 \%$, and $92 \%$ versus $77 \%$, respectively). A possible explanation for this difference was the patient's discomfort as a result of the additional instrumentation needed to remove tissue in the electrosurgical resection. However, in our study there were no outpatient procedures, which is why we think that these differences do not exist. A limitation of our study has been not long-term following up with the patients who underwent surgery in order to evaluate the efficacy of the resection and the recurrence rate between both groups.

Our study showed that hysteroscopic morcellation is faster and an equally effective alternative to bipolar resectoscopy in the hysteroscopic treatment of endometrial polyps. We have set out a future trial to compare hysteroscopic morcellation with resectoscopy for other types of intrauterine pathology.

\section{Financing}

The present investigation has not received specific grants from agencies of the public sector, commercial sector or nonprofit entities.

\section{Conflict of Interests}

The authors declare no conflict of interest.

\section{References}

1. Arnold A, Ketheeswaran A, Bhatti M, et al. (2016) A prospective analysis of hysteroscopic morcellation in the management of intrauterine pathologies. J Minim Invasive Gynecol 23: 435-441.

2. Cohen S, Greenberg JA (2011) Hysteroscopic morcellation for treating intrauterine pathology. Rev Obstet Gynecol 4: 73-80.

3. Hamerlynck TW, Schoot BC, van Vliet HA, et al. (2015) Removal of endometrial polyps: Hysteroscopic morcellation versus bipolar resectoscopy, a randomized trial. J Minim Invasive Gynecol 22: 1237-1243.

4. Smith PP, Middleton LJ, Connor M, et al. (2014) Hysteroscopic morcellation compared with electrical resection of endometrial polyps: A randomized controlled trial. Obstet Gynecol 123: 745751.

5. http://www.hesonline.nhs.uk/Ease/servlet/ContentServer?sitelD=1937\& categoryID=215.

6. Emanuel MH, Wamsteker K (2005) The Intra Uterine Morcellator: A new hysteroscopic operating technique to remove intrauterine polyps and myomas. J Minim Invasive Gynecol 12: 62-66.

7. Hamerlynck TW, Dietz V, Schoot BC (2011) Clinical implementation of the hysteroscopic morcellator for removal of intrauterine myomas and polyps. A retrospective descriptive study. Gynecol Surg 8: 193-196.

8. Tsuchiya A, Komatsu Y, Matsuyama R, et al. (2018) Intraoperative and postoperative clinical evaluation of the hysteroscopic morcellator system for endometrial polypectomy: A prospective, randomized, single-blind, parallel group comparison study. Gynecol Minim Invasive Ther 7: 16-21.

9. Li C, Dai Z, Gong Y, et al. (2017) A systematic review and metaanalysis of randomized controlled trials comparing hysteroscopic morcellation with resectoscopy for patients with endometrial lesions. Int J Gynaecol Obstet 136: 6-12.

10. Pampalona JR, Degollada BM, Mancebo MG, et al. (2015) Outpatient hysteroscopic polypectomy: Bipolar Energy System (Versapoint ${ }^{\circledR}$ ) versus Mechanical Energy System (TRUCLEAR System $^{\circledast}$ ) - Preliminary results. Gynecol Obstet Invest 80: 3-9.

11. Hamidouche A, Vincienne $M$, Thubert $T$, et al. (2015) Hysteroscopic morcellation versus bipolar resection for endometrial polyp removal. Gynecol Obstet Fertil 43: 104-108.

DOl: $10.36959 / 909 / 468$

Copyright: (C) 2019 López-Carral JM, et al. This is an open-access article distributed under the terms of the Creative Commons Attribution License, which permits unrestricted use, distribution, and reproduction in any medium, provided the original author and source are credited. 